\title{
Emerging chiral edge states from the confinement of a magnetic Weyl semimetal in $\mathrm{Co}_{3} \mathrm{Sn}_{2} \mathrm{~S}_{2}$
}

\author{
Lukas Muechler, ${ }^{1}$ Enke Liu, ${ }^{2,3}$ Jacob Gayles, ${ }^{2}$ Qiunan Xu, ${ }^{2}$ Claudia Felser, ${ }^{2, \text { * }}$ and Yan Sun ${ }^{2}$, \\ ${ }^{1}$ Center for Computational Quantum Physics, The Flatiron Institute, New York, New York, 10010, USA \\ ${ }^{2}$ Max Planck Institute for Chemical Physics of Solids, D-01187 Dresden, Germany \\ ${ }^{3}$ Institute of Physics, Chinese Academy of Sciences, Beijing 100190, China
}

(Dated: March 26, 2020)

\begin{abstract}
The quantum anomalous Hall effect (QAHE) and magnetic Weyl semimetals (WSMs) are topological states induced by intrinsic magnetic moments and spin-orbit coupling. Their similarity suggests the possibility of achieving the QAHE by dimensional confinement of a magnetic WSM along one direction. In this study, we investigate the emergence of the QAHE in the two-dimensional (2D) limit of magnetic WSMs due to finite size effects in thin films and step-edges. We demonstrate the feasibility of this approach with effective models and real materials. To this end, we have chosen the layered magnetic WSM $\mathrm{Co}_{3} \mathrm{Sn}_{2} \mathrm{~S}_{2}$, which features a large anomalous Hall conductivity and anomalous Hall angle in its $3 \mathrm{D}$ bulk, as our material candidate. In the $2 \mathrm{D}$ limit of $\mathrm{Co}_{3} \mathrm{Sn}_{2} \mathrm{~S}_{2}$ two QAHE states exist depending on the stoichiometry of the $2 \mathrm{D}$ layer. One is a semimetal with a Chern number of 6 , and the other is an insulator with a Chern number of 3 . The latter has a band gap of $0.05 \mathrm{eV}$, which is much larger than that in magnetically doped topological insulators. Our findings naturally explain the existence of chiral states in step edges of bulk $\mathrm{Co}_{3} \mathrm{Sn}_{2} \mathrm{~S}_{2}$ which habe been reported in a recent experiment at $T=4 K$ and present a realistic avenue to realize $\mathrm{QAH}$ states in thin films of magnetic WSMs.
\end{abstract}

\section{INTRODUCTION}

The quantum anomalous Hall effect (QAHE) is the quantized version of the anomalous Hall effect (AHE). In the QAHE, the transverse anomalous Hall conductance (AHC) is quantized in units of $e^{2} / h$ and the longitudinal resistance is reduced to zero 14 . In contrast to the ordinary AHE, the QAHE is topologically protected, and is characterized by a non-zero Chern number $n$ and chiral edge states in the bulk band gap. Contrary to the quantum Hall effect, which features the formation of Landau levels induced by an external magnetic field, the QAHE originates from intrinsic magnetic moments and spin orbit coupling (SOC). Because of the absence of back scattering, the electron currents carried by the edge states cannot be dissipated ${ }^{5}$. Therefore, realization of the QAHE and the utilization of its chiral edge state may lead to new low energy consumption quantum electronic devices. It was further proposed that interfaces of QAHE states and superconductors can generate chiral Majarona Fermions (MFs) ${ }^{6}$ via the proximity effect. These MFs are thought to be a fundamental ingredient for the development of topological quantum computing.

The QAHE was first proposed in 1988 by F. D. M. Haldane via a theoretical model defined on a honeycomb lattice ${ }^{4}$. However, because of the special requirements of this model, the realization of the QAHE in real materials took more than twenty years to achieve. Thanks to developments in topological band theory and thin film growth techniques, the effect was observed in counterparts of the QAHE, the quantum spin Hall effect (QSHE) and topological insulators (TIs). The first QAHE was observed in chromium-doped $(\mathrm{Bi}, \mathrm{Sb})_{2} \mathrm{Te}_{3}$, where the TI gains magnetic order through Van Vleck paramagnetism ${ }^{78}$. Based on the chiral edge states orig- inating from $\mathrm{Cr}$-doped $(\mathrm{Bi}, \mathrm{Sb})_{2} \mathrm{Te}_{3}$, chiral MFs were recently observed in the heterostructure of QAHE states and superconductors, which created strong enthusiasm for the comprehensive study of the QAHE and MFs using both theoretical and experimental approaches ${ }^{9}[13$.

Besides magnetically doped topological insulators ${ }^{78 / 14}$, much effort was also devoted to the search for new QAHE materials based on different mechanisms 10 13, such as magnetic and antiferromagnetic semiconductors 15 17, heterostructures of topological insulators (TIs) and ferromagnetic insulators 1823 heterostructures of heavy atom layers and magnets 2428 , honeycomb lattice (like graphen, silicene, and transition metal oxide with perovskite structure and pyrochlore oxide along (111) direction) with proximity coupling to ferromagnetic and antiferromagnetic insulators 29422 , asl well as thin films of 3D magnetic toplogical states $\frac{43}{3}$, and absorption of atoms on quantum spin Hall insulators $\$ 4$, etc. For example, a gap around $100 \mathrm{meV}$ was observed by ARPES in the heterostructure of $\mathrm{MnBi}_{2} \mathrm{Se}_{4} / \mathrm{Bi}_{2} \mathrm{Se}_{3}$.

Recently, another topological metallic state, the Weyl semimetal (WSM), was experimentally determined 46/47. Magnetic WSM with layered structures can be understood as coupled 2D QAHE layers 48149 . Motivated by this understanding and the development of WSMs, we propose another method of efficiently realizing the QAHE state in a real material. In WSMs, the conduction and valence bands linearly touch each other in 3D momentum space at Weyl points, which behave as monopoles of Berry curvatures with positive and negative chirality. According to symmetries, WSMs can be classified into two groups, those with and without time reversal symmetry. Since the Berry curvature is odd under time reversal, the magnetic WSMs often host a strong AHE originating from the Weyl points. Since 
Weyl points can only be defined in 3D momentum space, they require translation symmetry in 3D. Therefore, the Weyl point can be removed by opening a band gap through the breaking of periodic boundary conditions along one direction. If band ordering is maintained during this procedure, it is possible to obtain a QAHE phase in the 2D limit of the magnetic WSMs. On the other hand, since the QAHE exists only in the extreme 2D limit, magnetic WSMs with layered crystal structures are preferred material candidates.

\section{OUTLINE}

The paper is organized as follows: In Sec. III we review the proposal to realize a QAH state in thin films of a magnetic WSM based on model calculations. Using abinitio calculations, we show in Sec. IV that thin films of the magnetic WSM $\mathrm{Co}_{3} \mathrm{Sn}_{2} \mathrm{~S}_{2}$ are ideal candidates to realize the $\mathrm{QAH}$ state in two different stochiometries. In the last section (Sec. V), we discuss how these findings explain the recent observation of chiral modes on step edges of bulk $\mathrm{Co}_{3} \mathrm{Sn}_{2} \mathrm{~S}_{2}$.

\section{MODEL DISCUSSION}

To evaluate the feasibility of this proposal, we first analyzed the 2D limit of the Weyl semimetal using a toy two band model, $H=A\left(k_{x} \sigma(x)+k_{y} \sigma(y)\right)+M(k) \sigma_{z}$,

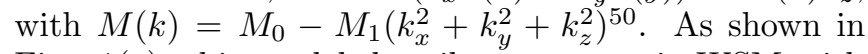
Fig. 1(a), this model describes a magnetic WSM with one pair of Weyl points located at $\pm k_{W}$ along the $k_{z^{-}}$ axis and the Fermi level $\left(\mathrm{E}_{F}\right)$ lies just at the Weyl point. Owing to the Berry flux between this pair of Weyl points, the anomalous Hall conductivity reaches a peak value at $\mathrm{E}_{F}$ with $\sigma_{A H}=\left(k_{w} / \pi\right)\left(e^{2} / h\right)$, which is proportional to the separation of the Weyl points, see Fig. 1 (b) and (c) 50 . Breaking the periodic boundary condition in the $z$ direction, the Weyl points are removed by the opening of the band gap. Depending on the interaction strength between the top and bottom sides of the film, the band order can change as the thickness varies, resulting in a quantized jump of the AHC from zero to a finite number. From the parameters specified in this study, the interactions between the top and bottom surfaces remove the band inversion of the 3D bulk state for thicknesses of one and two unit cells. Upon increasing the film thickness above three unit cells, the quantum confinement effect weakens and the band inversion remains, which leads to a quantized $\mathrm{AHC}$ and chiral edge state in the band gap, as shown in Fig. 1(df). Therefore, the mechanism for obtaining the QAHE from magnetic WSMs is principally allowed, but its realization from available materials remains challenging.

To date, many theoretical magnetic WSMs have been proposed, some of which feature strong AHEs 48|51|54.
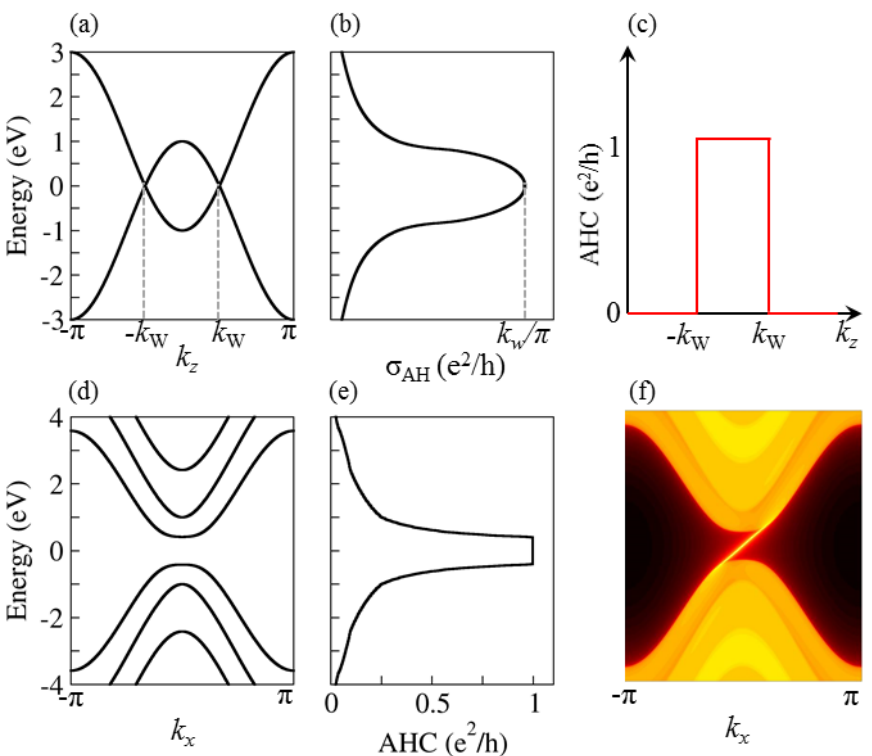

FIG. 1. (a) Energy dispersion along $k_{z}$ and (b) energy dependent anomalous Hall conductivity in the WSM effective model. (c) A quantized 2D-anomalous Hall conductance (AHC) exists between a pair of Weyl points for fixed $k_{z}$ between $-k_{w}<k_{z}<k_{w}$. (d) Energy dispersion of the film from the WSM effective model with a thickness of three unit cells. (e) The anomalous Hall conductance (AHC) is quantized in the band gap of the film. (f) Energy dispersion of the edge states of the film. We have projected the $k \cdot p$ model to a cubic lattice with a lattice constant of $1 \AA$, and parameters of $A=1.0 \mathrm{eV} \AA, M_{0}=1 \mathrm{eV}$, and $M_{1}=1 \mathrm{eV} \AA^{2}$

Since the QAHE is a 2D insulator, WSMs with large AHE, low charge carrier density, and layered lattice structure are preferred. In this study, we choose the experimentally well characterized quasi-2D material $\mathrm{Co}_{3} \mathrm{Sn}_{2} \mathrm{~S}_{2}$ to examine the possibility of realizing a QAHE based on a magnetic WSM. $\mathrm{Co}_{3} \mathrm{Sn}_{2} \mathrm{~S}_{2}$ is a half metal with a rhombohedral lattice structure in the space group $R \overline{3} m$ (No. 166) 55 57. The magnetic Co atoms are arranged in a Kagome lattice in the $x-y$ plane with magnetic moments along the $z$ axis. When the lattice is placed in a hexagonal setting, $\mathrm{Co}_{3} \mathrm{Sn}$ forms a quasi-2D layer and a sandwich between the $\mathrm{S}$ atoms. The quasi-2D layer $\mathrm{Co}_{3} \mathrm{SnS}_{2}$ are connected by another $\mathrm{Sn}$ atom in the $z$ direction, as shown in Fig. 2(a).

\section{SINGLE LAYER LIMIT}

Recently, large anomalous Hall conductivities up to $1100 \mathrm{~S} / \mathrm{cm}$ were observed in $\mathrm{Co}_{3} \mathrm{Sn}_{2} \mathrm{~S}_{2}$, originating from the SOC induced node-line-like band anticrossings and Weyl points, see Fig. 2(c, d). Owing to the low charge carrier density, the anomalous Hall angle reaches as high as $20 \%$, which has not been observed in other compounds 58159 . In this material, the Weyl points are very close to $\mathrm{E}_{F}$ and there is almost no interference from triv- 
(a)

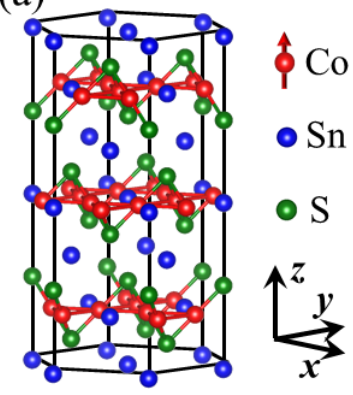

(c)

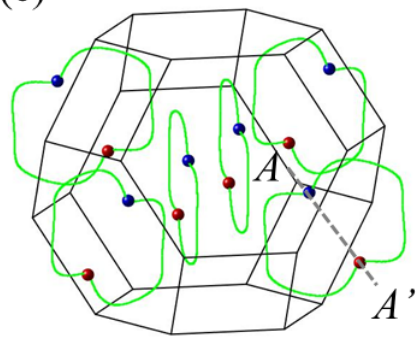

(b)

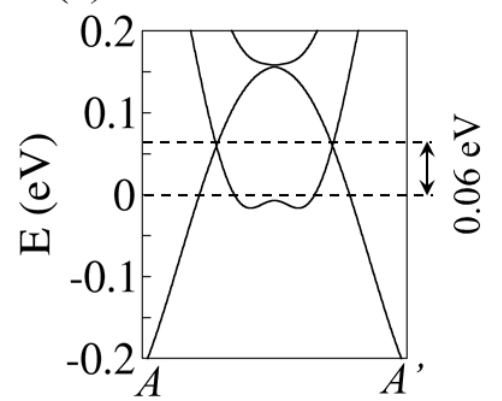

(d)

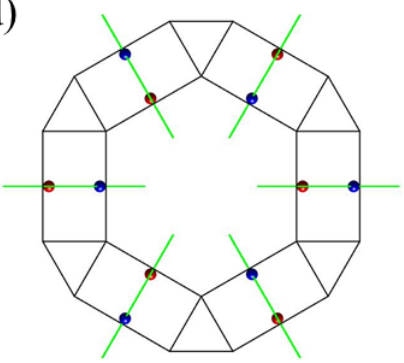

(a)

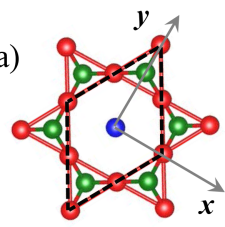

(b)

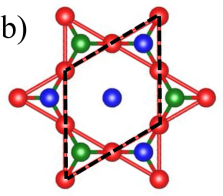

(c)

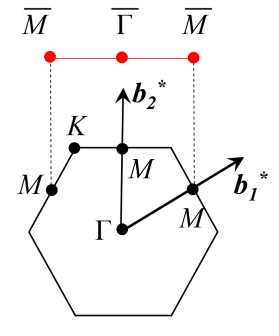

(d)
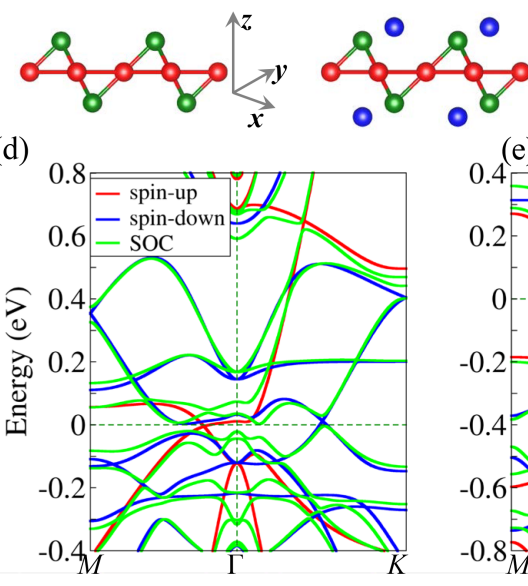

(e)

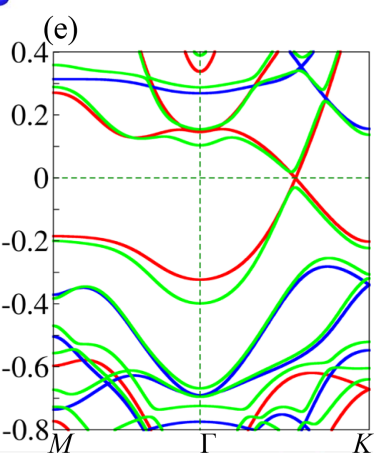

FIG. 2. (a) Lattice structure of $\mathrm{Co}_{3} \mathrm{Sn}_{2} \mathrm{~S}_{2}$. The spin polarization of the Co atom is along the $z$ direction. (b) Energy dispersion of $\mathrm{Co}_{3} \mathrm{Sn}_{2} \mathrm{~S}_{2}$ along one pair of Weyl points with opposite chirality. The labels for the $k$ points are given in (c). (c, d) Side view and top view of the nodal lines and Weyl points distribution in BZ, respectively. The red and blue dots represent opposite chirality of the Weyl points.

ial bands, in contrast to other proposed magnetic WSMs. These properties make $\mathrm{Co}_{3} \mathrm{Sn}_{2} \mathrm{~S}_{2}$ an ideal candidate to realize a $\mathrm{QAH}$ state with chiral edges modes in the $2 \mathrm{D}$ limit due to finite size effects. Because of the weak bonding between $\mathrm{Sn}$ and $\mathrm{Co}_{3} \mathrm{SnS}_{2}$ layers, it should be possible to achieve the $2 \mathrm{D}$ films and step states by breaking the weak bonds. Therefore, $\mathrm{Co}_{3} \mathrm{Sn}_{2} \mathrm{~S}_{2}$ provides an ideal platform to study the interplay between the QAHE and WSMs experimentally.

Two possible terminations exist for cleaved $\mathrm{Co}_{3} \mathrm{Sn}_{2} \mathrm{~S}_{2}$, one with a Sn termination and the other with a $\mathrm{S}$ termination. The Co atoms form a Kagome lattice, with the $\mathrm{Sn}$ atom in the center. Sandwiching the Kagome lattice of $\mathrm{Co}_{3} \mathrm{Sn}$ between two $\mathrm{S}$ layers, a minimized thickness can be obtained for the S-terminated $\mathrm{Co}_{3} \mathrm{SnS}_{2}$ film, see Fig. 3(a). By further sandwiching the $\mathrm{Co}_{3} \mathrm{SnS}_{2}$ with neighboring Sn layers, the other minimum thickness can be obtained for the Sn-terminated $\mathrm{Co}_{3} \mathrm{Sn}_{3} \mathrm{~S}_{2}$ film. To obtain the magnetic ground state, the total energies of magnetization along the (001), (100), (010), and (110) directions were compared for both the bulk state and the two possible structures for the monolayers via density functional theory (DFT) calculations 6061. For the bulk state, the total energy in (001) phase is around $1.4 \mathrm{meV}$ lower than that with magnetization oriented in the $x-y$ plane, which is consistent with the experimental measurements $56 / 58 / 59$. Similarly, the two monolayer struc-

FIG. 3. Lattice structure of (a) $\mathrm{Co}_{3} \mathrm{SnS}_{2}$ and (b) $\mathrm{Co}_{3} \mathrm{Sn}_{3} \mathrm{~S}_{2}$ films from the top (upper panel) and side (lower panel) view. (c) Corresponding 2D BZ and its $1 \mathrm{D}$ projection. The energy dispersion along high symmetry lines as shown for (d) $\mathrm{Co}_{3} \mathrm{SnS}_{2}$ and (e) $\mathrm{Co}_{3} \mathrm{Sn}_{3} \mathrm{~S}_{2}$ with and without the inclusion of SOC.

tures also preferred a magnetic structure with magnetization along the (001) direction, however the energy differences are only around 0.4 and $0.1 \mathrm{meV}$ in $\mathrm{Co}_{3} \mathrm{Sn}_{3} \mathrm{~S}_{2}$ and $\mathrm{Co}_{3} \mathrm{SnS}_{2}$ films, respectively, much lower than in the bulk with $T_{c} \simeq 200 \mathrm{~K}$. Owing to the $2 \mathrm{D}$ magnetic structure and the small anisotropic energy, the dipole-dipole iteraction might play an important role for the spin oriented in plane. However, the dipole-dipole interaction is around one order of magnitude weaker than the anisotropic energy, due to the small moment of the Co ions. see Fig. 4. Therefore, the magnetic are expected to along (001) direction. Based on the anisotropic energy, the magnetic order temperature for $\mathrm{Co}_{3} \mathrm{Sn}_{3} \mathrm{~S}_{2}$ and $\mathrm{Co}_{3} \mathrm{SnS}_{2}$ freestanding monolayers are around 5.0 and $1.0 \mathrm{~K}$, respectively. The anisotropy can increase in the presence of a substrate, which in turn can increase the ordering temperature.

Without SOC, the $\mathrm{Co}_{3} \mathrm{SnS}_{2}$ film shows a semi-metallic band structure. In contrast to the half-metallic state in the 3D bulk, both spin-up and spin-down channels appear close to the Fermi level in the $\mathrm{Co}_{3} \mathrm{SnS}_{2}$ films. Owing to the mirror planes of $M_{x}$ and $M_{y}$, both spinup and spin-down channels form the linear band crossing around $E_{F}$, which exist on the high symmetry lines along $M-\Gamma$ and $\Gamma-K$, respectively, see Fig. 3(d). After SOC is considered, the $\mathrm{SU}(2)$ symmetry is broken, leading to gapping of the linear band crossings.

Viewing the energy dispersion in a larger energy window, it is clear that another linear band exists crossing 
TABLE I. The comparison of total energies with magnetic polarization along (001) and other directions. The energy differences are in the unit of of $\mathrm{meV} /($ formula unit).

\begin{tabular}{cccc}
\hline \hline & $\mathrm{E}_{t o t}(100)-\mathrm{E}_{\text {tot }}(001)$ & $\mathrm{E}_{t o t}(010)-\mathrm{E}_{t o t}(001)$ & $\mathrm{E}_{t o t}(110)-\mathrm{E}_{t o t}(001)$ \\
\hline $\mathrm{Co}_{9} \mathrm{Sn}_{6} \mathrm{~S}_{6}(\mathrm{bulk})$ & 1.4 & 1.4 & 1.4 \\
$\mathrm{Co}_{3} \mathrm{Sn}_{3} \mathrm{~S}_{2}$ & 0.5 & 0.4 & 0.4 \\
$\mathrm{Co}_{3} \mathrm{SnS}_{2}$ & 0.2 & 0.2 & 0.1 \\
\hline \hline
\end{tabular}

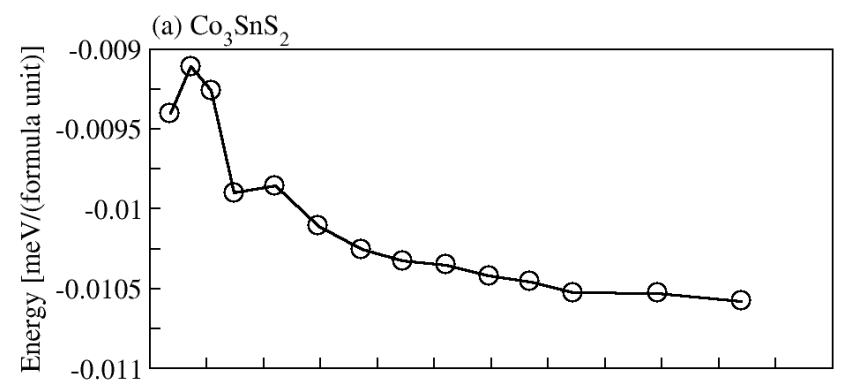

(b) $\mathrm{Co}_{3} \mathrm{Sn}_{3} \mathrm{~S}_{2}$

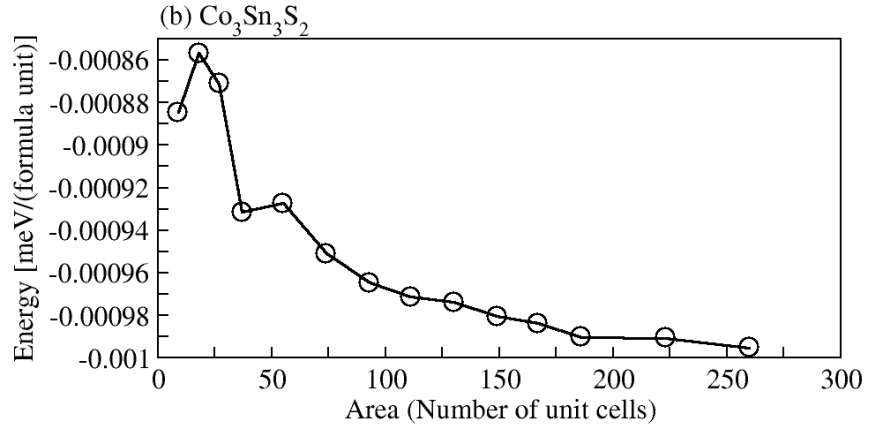

FIG. 4. Convergence of the dipole-dipole interaction along with the increasing of number of in-plane unit cells.

$\Gamma-K$ with its chemical potential shifting up by approximately $0.6 \mathrm{eV}$ and 4 electrons, corresponding to the filling expected for films with Sn termination. This system should act as a semi-metal from the perspective of transport, by simply shifting the chemical potential to the upper linear band crossing point, see Fig. 3(d). However, the Sn layers affect the filling and change the dispersion of the bands around $E_{F}$. As shown in Fig. 3 (e), after further sandwiching by Sn layers, the system has an ideal semi-metallic state with the Fermi surface composed solely of the linear crossing points. Similar to $\mathrm{Co}_{3} \mathrm{SnS}_{2}$, this linear band crossing is broken by SOC with the opening of a band gap. Compared to $\mathrm{Co}_{3} \mathrm{SnS}_{2}$, the $\mathrm{Sn}$ terminated film has a global band gap at approximately $0.05 \mathrm{eV}$, which is much larger than that in magneticimpurity-doped TIs.

To confirm the quantization of the anomalous Hall conductance (AHC), we calculated the energy dependent AHC by integrating the Berry curvature over the whole BZ. The AHC for $\mathrm{Co}_{3} \mathrm{SnS}_{2}$ can reach up to almost $6 e^{2} / h$ at the charge neutral point. However, since $\mathrm{Co}_{3} \mathrm{SnS}_{2}$ does not have a global band gap, its AHC changes sharply as the energy varies around $E_{F}$, and the maximum value only appears at an energy point, see Fig. 5(a). Though

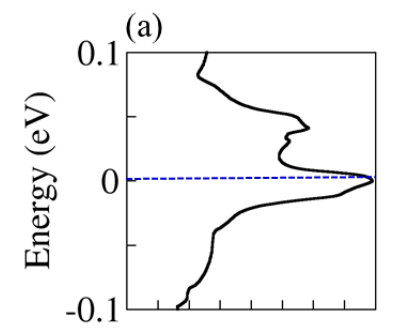

(b)
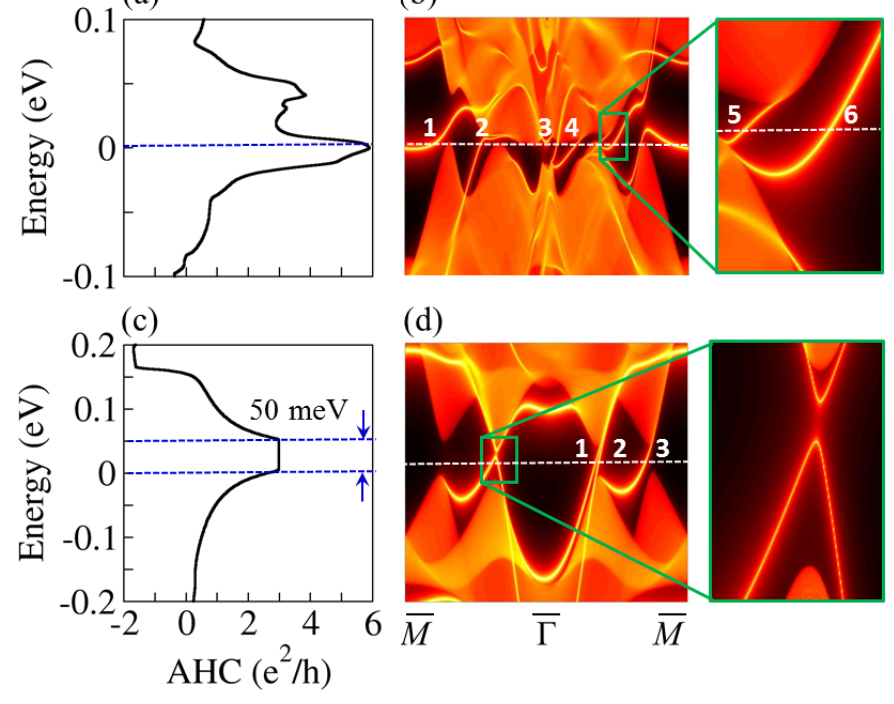

(d)

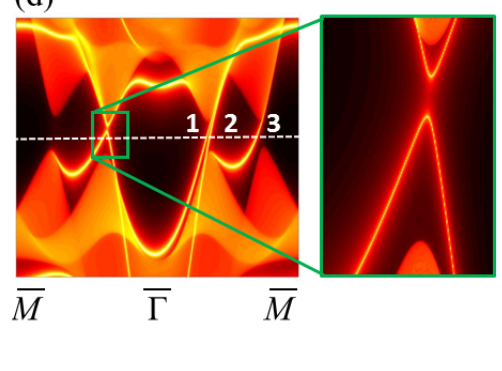

FIG. 5. (a, b) Energy dependent $\mathrm{AHC}$ for the $\mathrm{Co}_{3} \mathrm{SnS}_{2}$ film. The peak value with $\mathrm{AHC}=6 e^{2} / h$ appears at a charge neutral point. (b) Energy dispersion of the edge states for the $\mathrm{Co}_{3} \mathrm{SnS}_{2}$ film. The local energy dispersion around edge states No.5 and No.6 are given on the right. (c) Quantized $\mathrm{AHC}\left(3 e^{2} / h\right)$ appears in the band gap for the $\mathrm{Co}_{3} \mathrm{Sn}_{3} \mathrm{~S}_{2}$ film. (d) Edge states of $\mathrm{Co}_{3} \mathrm{Sn}_{3} \mathrm{~S}_{2}$ film. The trivial dangling bond states are zoomed in on the right side.

$\mathrm{Co}_{3} \mathrm{Sn}_{3} \mathrm{~S}_{2}$ has four more electrons than $\mathrm{Co}_{3} \mathrm{SnS}_{2}$, only three of them have non-zero chern number $(-1)$, leading to the Chern number jump from 6 to 3 . In contrast the the semimetallic state in $\mathrm{Co}_{3} \mathrm{SnS}_{2}$, the film with a stoichiometry of $\mathrm{Co}_{3} \mathrm{Sn}_{3} \mathrm{~S}_{2}$ has a constant quantized $\mathrm{AHC}$ in the energy range from $E_{F}$ to $E_{F}+50 \mathrm{meV}$, as shown in Fig. 5(c). Thus, both the $\mathrm{Co}_{3} \mathrm{SnS}_{2}$ and $\mathrm{Co}_{3} \mathrm{Sn}_{3} \mathrm{~S}_{2}$ films host nontrivial topological electronic structures with nonzero Chern numbers of 6 and 3, respectively.

A typical feature of the quantum anomalous Hall insulators and semi-metals is their chiral edge state. To calculate the edge states, we projected the Bloch wave functions into Wannier orbitals 62, and constructed a tight binding model Hamiltonian. The edge state was considered under open boundary conditions with the halfinfinite one-dimensional model using the iterative Greens

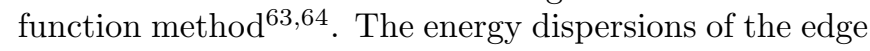
states for films with the nominal compositions $\mathrm{Co}_{3} \mathrm{SnS}_{2}$ and $\mathrm{Co}_{3} \mathrm{Sn}_{3} \mathrm{~S}_{2}$ are shown in Fig. 5(b) and (d), respectively. Due to the semi-metallic properties of the mono- 
layers with the stoichiometry $\mathrm{Co}_{3} \mathrm{SnS}_{2}$, the projections of the bulk conduction and valence are separated by a gap, which are connected by six edge bands. The edge bands all posses a positive Fermi velocity, corresponding to a Chern number of 6 . In the monolayers with the stoichiometry $\mathrm{Co}_{3} \mathrm{Sn}_{3} \mathrm{~S}_{2}$, three topological protected channels exist on the edge with positive Fermi velocity, which is consistent with a Chern number of 3 obtained from the calculation of the quantized AHC, see Fig. 5(d). Since the properties of the edge states are strongly dependent on the nature of the edge termination, the topological edge-states can be distinguished from trivial dangling bond states, since the dangling bonds are not topologically protected. They do not connect the valence and conduction states of the bulk, as can bee seen in the zoom in in Fig. 5(d), and should be easily annihilated by chemical engineering of the edge termination.

\section{CHIRAL MODES AT STEP EDGES}

Although a QAHE is expected in monolayers of the stoichiometry $\mathrm{Co}_{3} \mathrm{SnS}_{2}$ and $\mathrm{Co}_{3} \mathrm{Sn}_{3} \mathrm{~S}_{2}$, their free standing form is not expected to be stable without a substrate, indicated by phonon frequencies of the free standing slabs. A suitable substrate could stabilize these monolayers at higher temperatures due to kinetic effects, however this is beyond the possibilities of DFT to predict and requires additional experimental input. However, our results further imply the presence of chiral modes at step edges on bulk $\mathrm{Co}_{3} \mathrm{Sn}_{2} \mathrm{~S}_{2}$, which could be easily measured by STM experiments. A step edge can be modeled as a single QAH layer that is coupled to the bulk WSM. If the coupling between the QAH layer and the bulk can be treated perturbatively as expected in a layered material, the step edge is expected to host chiral edge states, similarly to the QSH step edge states observed in $\mathrm{WTe}_{2}{ }^{65}$. Even in the case of stronger coupling between the layers, the 'terrace construction' of Hsieh et al. ${ }^{[66}$ suggests the presence of spatially separated, counterpropagating chiral states at step edges that could be observed in STM experiments, as the combined Chern number of the top layer and WSM has to vanish. In this setup, one would expect to observe the edge states at temperatures close to the bulk $T_{c} \simeq 200 K$, which could open a pathway to more detailed studies of topological chiral states at higher temperatures. Indeed, such states have recently been reported in STM experiments on step-edges at $T=4 K^{\sqrt{67}}$ as a direct consequence of the topological non-trivial electronic structure in the thin film limit.

\section{SUMMARY}

In summary, we theoretically studied the QAHE from the quantum confinement of the magnetic WSMs. Since the WSM can only be defined in 3D momentum space, a method for the phase transition from WSM to QAHE is breaking the translational symmetry along one direction in the WSM. Considering the 2D topological insulating state in the QAHE, the WSMs with strong AHE, low charge density, and layered lattice structure are preferred candidates. Considering these material requirements, we focused on the layered magnetic WSM $\mathrm{Co}_{3} \mathrm{Sn}_{2} \mathrm{~S}_{2}$ as a candidate for the realization of QAHE from WSMs. In the 2D limit of $\mathrm{Co}_{3} \mathrm{Sn}_{2} \mathrm{~S}_{2}$, we find two possible QAHE states. One is a semi-metal with a Chern number of 6 , and the other is an insulator with a Chern number of 3 and band gap of $0.05 \mathrm{eV}$. Our findings are supported by the recent observation of chiral modes at step edges of bulk $\mathrm{Co}_{3} \mathrm{Sn}_{2} \mathrm{~S}_{2}$, which follows from the non-zero Chern numbers in the $2 \mathrm{D}$ limit of $\mathrm{Co}_{3} \mathrm{Sn}_{2} \mathrm{~S}_{2} 6$.

\section{ACKNOWLEDGMENTS}

This work was financially supported by the ERC Advanced Grant No. 291472 'Idea Heusler', ERC Advanced Grant No. 742068-TOPMAT, and Deutsche Forschungsgemeinschaft DFG under SFB 1143. EL acknowledges support from the Alexander von Humboldt foundation of Germany for his Fellowship and from National Natural Science Foundation of China for his Excellent Young Scholarship (No. 51722106). LM would like to thank the MPI CPFS where part of the work was performed. and the authors thank Binghai Yan for helpful discussions. The Flatiron Institute is a division of the Simons Foundation.
* Claudia.Felser@cpfs.mpg.de

†ysun@cpfs.mpg.de

1 E. H. Hall, Amer. J. Math. 2, 287 (1879).

2 E. H. Hall, The London, Edinburgh, and Dublin Philosophical Magazine and Journal of Science 12, 157 (1881).

3 N. Nagaosa, J. Sinova, S. Onoda, A. H. MacDonald, and N. P. Ong, Rev. Mod. Phys. 82, 1539 (2010).

${ }^{4}$ F. D. M. Haldane, Phys. Rev. Lett. 61, 2015 (1988).

5 B. I. Halperin, Phys. Rev. B 25, 2185 (1982).
${ }^{6}$ X.-L. Qi, T. L. Hughes, and S.-C. Zhang, Phys. Rev. B 82, 184516 (2010).

7 R. Yu, W. Zhang, H.-J. Zhang, S.-C. Zhang, X. Dai, and Z. Fang, Science 329, 61 (2010).

8 C.-Z. Chang, J. Zhang, X. Feng, J. Shen, Z. Zhang, M. Guo, K. Li, Y. Ou, P. Wei, L.-L. Wang, et al., Science 340, 167 (2013).

${ }^{9}$ Q. L. He, L. Pan, A. L. Stern, E. C. Burks, X. Che, G. Yin, J. Wang, B. Lian, Q. Zhou, E. S. Choi, et al., Science 357, 
294 (2017).

10 C.-X. Liu, S.-C. Zhang, and X.-L. Qi, Annual Review of Condensed Matter Physics 7, 301 (2016).

11 H. Weng, R. Yu, X. Hu, X. Dai, and Z. Fang, Advances in Physics 64, 227 (2015).

12 Y. Ren, Z. Qiao, and Q. Niu, Reports on Progress in Physics 79, 066501 (2016).

13 J. Wang, B. Lian, and S.-C. Zhang, Physica Scripta 2015, $014003(2015)$.

14 C.-X. Liu, X.-L. Qi, X. Dai, Z. Fang, and S.-C. Zhang, Phys. Rev. Lett. 101, 146802 (2008).

15 Q.-F. Liang, L.-H. Wu, and X. Hu, New Journal of Physics 15, 063031 (2013).

16 J. He, X. Li, P. Lyu, and P. Nachtigall, Nanoscale 9, 2246 (2017).

17 X.-L. Sheng and B. K. Nikoli, Phy. Rev. B 95, 201402(R) (2017).

18 S. Eremeev, V. Men'Shov, V. Tugushev, P. M. Echenique, and E. V. Chulkov, Phys. Rev. B 88, 144430 (2013).

19 S. Eremeev, V. Men, V. Tugushev, E. V. Chulkov, et al., J. Magn. Magn. Mater. 383, 30 (2015).

${ }^{20}$ F. Katmis, V. Lauter, F. S. Nogueira, B. A. Assaf, M. E. Jamer, P. Wei, B. Satpati, J. W. Freeland, I. Eremin, D. Heiman, et al., Nature 533, 513 (2016).

21 M. M. Otrokov, T. V. Menshchikova, M. G. Vergniory, I. P. Rusinov, A. Y. Vyazovskaya, Y. M. Koroteev, G. Bihlmayer, A. Ernst, P. M. Echenique, A. Arnau, et al., 2D Materials 4, 025082 (2017).

22 M. Otrokov, T. V. Menshchikova, I. Rusinov, M. Vergniory, V. M. Kuznetsov, and E. V. Chulkov, JETP Letters 105, 297 (2017).

23 J. A. Hagmann, X. Li, S. Chowdhury, S.-N. Dong, S. Rouvimov, S. J. Pookpanratana, K. M. Yu, T. A. Orlova, T. B. Bolin, C. U. Segre, et al., New Journal of Physics 19, 085002 (2017).

${ }^{24}$ K. F. Garrity and D. Vanderbilt, Phys. Rev. Lett. 110, 116802 (2013).

${ }^{25}$ K. F. Garrity and D. Vanderbilt, Phys. Rev. B 90, 121103 (2014).

26 J. Liu, S. Y. Park, K. F. Garrity, and D. Vanderbilt, Phys. Rev. Lett. 117, 257201 (2016).

27 G. Xu, J. Wang, C. Felser, X.-L. Qi, and S.-C. Zhang, Nano Lett. 15, 2019 (2015).

${ }^{28}$ L. Si, O. Janson, G. Li, Z. Zhong, Z. Liao, G. Koster, and K. Held, Phys. Rev. Lett. 119, 026402 (2017).

29 Z. Qiao, S. A. Yang, W. Feng, W.-K. Tse, J. Ding, Y. Yao, J. Wang, and Q. Niu, Phys. Rev. B 82, 161414 (2010).

30 R. Nandkishore and L. Levitov, Phys. Rev. B 82, 115124 (2010).

31 W.-K. Tse, Z. Qiao, Y. Yao, A. H. MacDonald, and Q. Niu, Phys. Rev. B 83, 155447 (2011).

${ }^{32}$ F. Zhang, J. Jung, G. A. Fiete, Q. Niu, and A. H. MacDonald, Phys. Rev. Lett. 106, 156801 (2011).

33 M. Ezawa, Phys. Rev. Lett. 109, 055502 (2012).

34 M. Ezawa, Phys. Rev. Lett. 110, 026603 (2013).

35 J. Zhang, B. Zhao, Y. Yao, and Z. Yang, Scientific reports 5, 10629 (2015).

36 J. Zhang, B. Zhao, Y. Yao, and Z. Yang, Phys. Rev. B 92, 165418 (2015).

37 Z. Qiao, W. Ren, H. Chen, L. Bellaiche, Z. Zhang, A. H. MacDonald, and Q. Niu, Phys. Rev. Lett. 112, 116404 (2014).

38 D. Xiao, W. Zhu, Y. Ran, N. Nagaosa, and S. Okamoto, Nat. Commun. 2, 596 (2011).
39 A. Rüegg and G. A. Fiete, Phys. Rev. B 84, 201103 (2011).

$40 \mathrm{X} . \mathrm{Hu}, \mathrm{A}$. Rüegg, and G. A. Fiete, Phys. Rev. B 86, 235141 (2012).

41 A. Zyuzin, S. Wu, and A. Burkov, Phys. Rev. B 85, 165110 (2012).

42 Q.-F. Liang, L.-H. Wu, and X. Hu, New Journal of Physics 15, 063031 (2013).

43 G. Xu, H. Weng, Z. Wang, X. Dai, and Z. Fang, Phys. Rev. Lett. 107, 186806 (2011).

44 S.-C. Wu, G. Shan, and B. Yan, Phys. Rev. Lett. 113, 256401 (2014).

45 T. Hirahara, S. V. Eremeev, T. Shirasawa, Y. Okuyama, T. Kubo, R. Nakanishi, R. Akiyama, A. Takayama, T. Hajiri, S.-i. Ideta, et al., Nano Lett. 17, 3493 (2017).

46 S.-Y. Xu, I. Belopolski, N. Alidoust, M. Neupane, G. Bian, C. Zhang, R. Sankar, G. Chang, Z. Yuan, C.-C. Lee, et al., Science 349, 613 (2015).

47 B. Lv, H. Weng, B. Fu, X. Wang, H. Miao, J. Ma, P. Richard, X. Huang, L. Zhao, G. Chen, et al., Physical Review X 5, 031013 (2015).

48 X. Wan, A. M. Turner, A. Vishwanath, and S. Y. Savrasov, Phys. Rev. B 83, 205101 (2011).

49 A. Burkov and L. Balents, Phys. Rev. Lett. 107, 127205 (2011).

50 H.-Z. Lu, S.-B. Z. Zhang, and S.-Q. Shen, Phy. Rev. B 92, 045203 (2015).

51 G. Xu, H. Weng, Z. Wang, X. Dai, and Z. Fang, Phys. Rev. Lett. 107, 186806 (2011).

52 Z. Wang, M. Vergniory, S. Kushwaha, M. Hirschberger, E. Chulkov, A. Ernst, N. P. Ong, R. J. Cava, and B. A. Bernevig, Phys. Rev. Lett. 117, 236401 (2016).

53 T.-R. Chang, G. Chang, C.-C. Lee, S.-M. Huang, B. Wang, G. Bian, H. Zheng, D. S. Sanchez, I. Belopolski, N. Alidoust, M. Neupane, A. Bansil, H.-T. Jeng, S.-Y. Xu, H. Lin, and M. Z. Hasan, Nat. Commun. 7, 1 (2016).

54 J. Kübler and C. Felser, EPL (Europhysics Letters) 114, 47005 (2016).

55 R. Weihrich, I. Anusca, and M. Zabel, ChemInform 36, 35 (2005).

56 P. Vaqueiro and G. G. Sobany, Solid State Sci. 11, 513 (2009).

57 W. Schnelle, A. Leithe-Jasper, H. Rosner, F. Schappacher, R. Pöttgen, F. Pielnhofer, and R. Weihrich, Phys. Rev. B 88, 144404 (2013).

58 E. Liu, Y. Sun, N. Kumar, L. Muechler, A. Sun, L. Jiao, S.-Y. Yang, D. Liu, A. Liang, Q. Xu, et al., Nat. Phys. 14, 1125 (2018).

59 Q. Wang, Y. Xu, R. Lou, Z. Liu, M. Li, Y. Huang, D. Shen, H. Weng, S. Wang, and H. Lei, Nat. Commun. 9, 3681 (2018).

60 G. Kresse and J. Furthmüller, Phys. Rev. B 54, 11169 (1996).

61 J. P. Perdew, K. Burke, and M. Ernzerhof, Phys. Rev. Lett. 77, 3865 (1996).

62 A. A. Mostofi, J. R. Yates, Y.-S. Lee, I. Souza, D. Vanderbilt, and N. Marzari, Comput. Phys. Commun 178, 685 (2008).

63 M. L. Sancho, J. L. Sancho, and J. Rubio, J. Phys. F Met. Phys. 14, 1205 (1984).

64 M. L. Sancho, J. L. Sancho, J. L. Sancho, and J. Rubio, J. Phys. F Met. Phys. 15, 851 (1985).

65 L. Peng, Y. Yuan, G. Li, X. Yang, J.-J. Xian, C.-J. Yi, Y.-G. Shi, and Y.-S. Fu, Nat. Commun. 8, 659 (2017). 
66 T. H. Hsieh, H. Ishizuka, L. Balents, and T. L. Hughes, Phys. Rev. Lett. 116, 086802 (2016)

67 S. Howard, L. Jiao, Z. Wang, P. Vir, C. Shekhar, C. Felser, T. Hughes, and V. Madhavan, arXiv preprint
arXiv:1910.11205 (2019) 